\title{
Nonequilibrium chiral magnetic/vortical effects in viscous fluids
}

\author{
Yoshimasa Hidaka ${ }^{1,2}$ and Di-Lun Yang ${ }^{1}$ \\ ${ }^{1}$ Nishina Center, RIKEN, Wako, Saitama 351-0198, Japan \\ ${ }^{2}$ iTHEMS Program, RIKEN, Wako, Saitama 351-0198, Japan
}

(Received 26 January 2018; revised manuscript received 10 April 2018; published 26 July 2018)

\begin{abstract}
We utilize the chiral kinetic theory in a relaxation-time approximation to investigate the nonlinear anomalous responses of chiral fluids with viscous effects. Unlike the cases in equilibrium, it is found that the chiral magnetic effect and chiral vortical effect are modified by the shear and bulk strengths. Particularly, the shear strength could result in charged Hall currents for chiral magnetic and chiral vortical effects, which propagate perpendicular to applied magnetic fields and vorticity. These quantum corrections stemming from side jumps and anomalies are dissipative and pertinent to interactions. Although the nonequilibrium effects upon charge currents are dissipative, the second law of thermodynamics is still satisfied.
\end{abstract}

DOI: 10.1103/PhysRevD.98.016012

\section{INTRODUCTION}

The anomalous transport for Weyl fermions related to quantum anomalies such as chiral magnetic/vortical effects (CME/CVE), from which charged currents are induced by magnetic/vortical fields, has recently aroused great interest in the studies of relativistic heavy ion collisions (HIC) and Weyl semimetals [1-4]. Such effects and relevant phenomena associated with chiral imbalance have been investigated from various approaches including fieldtheory calculations based on Kubo formula [3,5,6], kinetic theory [7-17], relativistic hydrodynamics [18-22], lattice simulations [23-29], and gauge/gravity duality [30-33]. Particularly, recent progress in chiral kinetic theory (CKT) with the manifestation of Lorenz symmetry related to side jumps and the incorporation of collisions has improved our understandings on anomalous transport out of equilibrium [34-36].

It is generally believed that $\mathrm{CME}$ in equilibrium is protected by the chiral anomaly and unaffected by interactions. On the other hand, CVE in equilibrium could be protected by mixed-axial-gravitational anomaly with only background fields $[6,33,37]$, whereas the modification from interactions could emerge in the presence of dynamical gauge fields [38-40]. See Refs. [41-43] for some following works. In addition, there are studies for the axial currents induced by electric fields and chiral imbalance such as the chiral electric separation effect (CESE) or chiral Hall effect (CHE), which are pertinent to interactions [44-46], while

Published by the American Physical Society under the terms of the Creative Commons Attribution 4.0 International license. Further distribution of this work must maintain attribution to the author(s) and the published article's title, journal citation, and DOI. Funded by SCOAP. these effects are not directly connected to the helicity of Weyl fermions and quantum anomalies. Nonetheless, an interplay between the chiral anomaly, magnetization current stemming from side jumps, and magnetic-moment coupling could result in nonequilibrium corrections involving interactions upon anomalous transport. For example, the alternative-current (AC) conductivity for $\mathrm{CME}$ with nonzero frequency is modified in the presence of timedependent magnetic fields $[5,14,47]$. Also, the nonlinear responses on anomalous transport triggered by fluctuations near local equilibrium in inviscid chiral fluids have been analyzed by some of the authors in this paper [48], where novel anomalous Hall currents led by electric fields and temperature/chemical-potential gradients are found [49]. However, the nonequilibrium corrections on charge currents from magnetic fields or vorticity may appear in the viscous case [52]. Experimentally, in HIC, due to the viscous corrections in the quark gluon plasma, the nonequilibrium effects upon the CME conductivity may impact the charge separation associated with the signal for CME. Moreover, based on recent observations of hydrodynamic transport in graphene [54,55], there exists mounting interest in the possible realization of chiral fluids in Weyl semimetals (see e.g., Refs. [56,57] for relevant studies in theory). The theoretical investigation of relativistic viscous hydrodynamics of chiral fluids is thus imperative.

In this paper, we follow the approach in Ref. [48] to further study the viscous corrections on anomalous transport contributed by the aforementioned quantum effects associated with the helicity of Weyl fermions. It is found that the CME and CVE conductivities of charge currents are modified by shear and bulk strengths. Nevertheless, the non-equilibrium corrections on the energy-density currents should vanish according to the matching condition in the classical relaxation-time approximation (RTA). Although 
the nonequilibrium quantum corrections are dissipative, the second law of thermodynamics is still satisfied.

\section{WIGNER FUNCTIONS AND CHIRAL KINETIC THEORY}

We begin with a brief introduction to the Wignerfunction formalism of CKT which will be exploited to study the nonequilibrium transport. As derived in Ref. [36] by solving Dirac equations up to $\mathcal{O}(\hbar)$ from the Wignerfunction approach, the perturbative solution for the less propagators of right-handed Weyl fermions reads

$$
\begin{aligned}
\grave{S}^{<\mu}(q, X)= & 2 \pi \bar{\epsilon}(q \cdot n)\left(q^{\mu} \delta\left(q^{2}\right)+\hbar \delta\left(q^{2}\right) S_{(n)}^{\mu \nu} \mathcal{D}_{\nu}\right. \\
& \left.+\hbar \epsilon^{\mu \nu \alpha \beta} q_{\nu} F_{\alpha \beta} \frac{\partial \delta\left(q^{2}\right)}{2 \partial q^{2}}\right) f_{q}^{(n)}
\end{aligned}
$$

where $\bar{\epsilon}(q \cdot n)$ represents the sign of $q \cdot n$ and

$$
S_{(n)}^{\mu \nu}=\frac{\epsilon^{\mu \nu \alpha \beta}}{2(q \cdot n)} q_{\alpha} n_{\beta}
$$

corresponds to the spin tensor depending on a frame vector $n^{\mu}$ [35]. The frame vector can be understood as the zeroth component of a vierbein for the local transformation of $\sigma^{a}=\left(I, \sigma^{i}\right)$ introduced on the local tangent space to $\sigma^{\mu}(X)$ depending on the global spacetime coordinates, where $\sigma^{i}$ represent Pauli matrices for $i=1,2,3$, such that $\sigma^{\mu}=e_{a}^{\mu} \sigma^{a}$. That is, we define $n^{\mu} \equiv e_{0}^{\mu}$ with $\mu$ being the spacetime indices (See Appendix A for details of the choice of the frame vector). In flat spacetime, we may set $e_{a}^{\mu}(X)=$ $\delta_{a}^{\mu}$ such that the spin connection vanishes. Now, the global spacetime coordinate transformation corresponds to the frame transformation $n^{\mu} \rightarrow n^{\prime \mu}$.

Here we denote $\mathcal{D}_{\beta} f_{q}^{(n)}=\Delta_{\beta} f_{q}^{(n)}-\mathcal{C}_{\beta}$, where $\Delta_{\mu}=$ $\partial_{\mu}+F_{\nu \mu} \partial_{q}^{\nu}, \quad \mathcal{C}_{\beta}=\Sigma_{\beta}^{<} \bar{f}_{q}^{(n)}-\Sigma_{\beta}^{>} f_{q}^{(n)} \quad$ with $\quad \Sigma_{\beta}^{<(>)}$being less/greater self-energies and $f_{q}^{(n)}$ and $\bar{f}_{q}^{(n)}=1-f_{q}^{(n)}$ being the distribution functions of incoming and outgoing particles, respectively. The second term in Eq. (1) associated with $S_{(n)}^{\mu \nu}$ as the side-jump term only contributes for the nonequilibrium cases or for a rotating system in global equilibrium, which results in magnetization currents and CVE. On the other hand, the third term in Eq. (1) yields $\mathrm{CME}$ in equilibrium.

Given the Wigner functions, one can directly evaluate the charge current and energy-momentum tensor through

$$
\begin{aligned}
T^{\mu \nu} & =\int \frac{d^{4} q}{(2 \pi)^{4}}\left(q^{\mu} \grave{S}^{<\nu}+q^{\nu} \grave{S}^{<\mu}\right), \\
J^{\mu} & =2 \int \frac{d^{4} q}{(2 \pi)^{4}} \grave{S}^{<\mu} .
\end{aligned}
$$

The distribution function in $\grave{S}^{<\mu}$ has to be solved from the CKT led by the Dirac equation. In light of the study in Ref. [48], we focus on the fluctuations slightly away from the local equilibrium distribution function defined in a comoving frame $n^{\mu}=u^{\mu}$ with $u^{\mu}$ being a fluid velocity.

For an arbitrary frame $n^{\mu}$, the CKT takes the form [48],

$$
\delta\left(q^{2}-\hbar \frac{B \cdot q}{q \cdot n}\right) \square(q, X) f_{q}^{(n)}=\delta\left(q^{2}-\hbar \frac{B \cdot q}{q \cdot n}\right) \mathcal{C}_{\text {full }},
$$

where

$$
\begin{aligned}
\square(q, X)= & {\left[q \cdot \Delta+\hbar \frac{S_{(n)}^{\mu \nu} E_{\mu}}{(q \cdot n)} \Delta_{\nu}+\hbar S_{(n)}^{\mu \nu}\left(\partial_{\mu} F_{\rho \nu}\right) \partial_{q}^{\rho}\right.} \\
& \left.+\hbar\left(\partial_{\mu} S_{(n)}^{\mu \nu}\right) \Delta_{\nu}\right]
\end{aligned}
$$

and

$$
\begin{aligned}
\mathcal{C}_{\text {full }} & =\left(q^{\mu}+\hbar \frac{S_{(n)}^{\nu \mu} E_{\nu}}{(q \cdot n)}+\hbar\left(\partial_{\rho} S_{(n)}^{\rho \mu}\right)\right) \tilde{\mathcal{C}}_{\mu}, \\
\tilde{\mathcal{C}}^{\mu} & =\mathcal{C}^{\mu}+\hbar \frac{\epsilon^{\mu \nu \alpha \beta} n_{\nu}}{2 q \cdot n}\left(\bar{f}_{q}^{(n)} \Delta_{\alpha}^{>} \Sigma_{\beta}^{<}-f_{q}^{(n)} \Delta_{\alpha}^{<} \Sigma_{\beta}^{>}\right) .
\end{aligned}
$$

Here, we define $B^{\mu}$ and $E_{\mu}$ by decomposing the field strength into $F_{\alpha \beta}=-\epsilon_{\mu \nu \alpha \beta} B^{\mu} n^{\nu}+n_{\beta} E_{\alpha}-n_{\alpha} E_{\beta}$. We will then work in $n^{\mu}=u^{\mu}$. The collision term depends on details of the system, so that it is not universal. Here, for simplicity and generality to be applicable for various systems including e.g., HIC or Weyl semimetals and also to make comparisons with previous studies [50,51] on equal footing, we apply the RTA for the collisional kernel in order to capture the qualitative features based on the symmetric properties of collisions. We thus approximate

$$
\mathcal{C}_{\text {full }} \simeq-\frac{1}{\tau_{R}}\left(q \cdot u+\hbar \frac{q^{\mu} \mathcal{A}_{\mu}}{(q \cdot u)^{2}}\right) \delta f_{q},
$$

where $\mathcal{A}_{\mu}$ denotes an operator acting on $\delta f_{q}$, which represents possible quantum corrections with $\mathcal{O}(\partial)$ that depends on the details of collision terms. $\tau_{R}$ is the relaxation time characterizing the inverse strength of interactions, which will be treated as a constant [58]. Note that here $\mathcal{A}_{\mu}$ is also frame dependent. The transformation of $\mathcal{A}_{\mu}$ in different frames should preserve the frame independence (Lorentz invariance) of the CKT with the RTA. We introduce such a frame transformation upon $\mathcal{A}_{\mu}$ in Appendix B.

We will then follow the computations in Ref. [48] to perturbatively solve for the non-equilibrium distribution function $\delta f_{q}=f_{q}^{(u)}-f_{q}^{\mathrm{eq}}$ incorporating viscous corrections from the CKT in Eq. (4) based on the $\hbar$ and derivatives expansions. Here the local equilibrium 
distribution function takes the form $f_{q}^{\mathrm{eq}}=\left(e^{g}+1\right)^{-1}$ with $g=\left(q \cdot u-\mu+\hbar(\omega \cdot q)(2 q \cdot u)^{-1}\right) / T$ for $T$ and $\mu$ being the local temperature and chemical potential, respectively. Also, the vorticity $\omega^{\mu}$ is defined as $\omega^{\mu} \equiv \epsilon^{\mu \nu \alpha \beta} u_{\nu}\left(\partial_{\alpha} u_{\beta}\right) / 2$.

\section{Hydrodynamics and matching conditions}

Furthermore, following the charge and energy-momentum conservation with the chiral anomaly, we should also implement the anomalous hydrodynamic EOM led by

$$
\partial_{\mu} J^{\mu}=-\frac{\hbar}{4 \pi^{2}} E_{\mu} B^{\mu}, \quad \partial_{\mu} T^{\mu \nu}=F^{\nu \mu} J_{\mu} .
$$

These two equations provide the physical constrains for CKT, which dictate the dynamics of thermodynamic parameters $T, \mu$, and $u^{\mu}$ in $f^{\text {eq }}$. However, by utilizing the equation $\Delta_{\mu} \grave{S}<\mu=\Sigma_{\mu}^{<} \grave{S}^{>\mu}-\Sigma_{\mu}^{>} \grave{S}^{<\mu}$ as the origin of the CKT, it is shown in Ref. [48] that the divergence of currents manifests the chiral anomaly,

$$
\begin{aligned}
\partial_{\mu} J^{\mu}= & -\frac{\hbar}{4 \pi^{2}} E_{\mu} B^{\mu} \\
& +2 \int_{q}\left[\delta\left(q^{2}\right) q^{\mu}+\hbar \epsilon^{\mu \nu \alpha \beta} F_{\alpha \beta} \frac{\partial_{q \nu} \delta\left(q^{2}\right)}{4}\right] \tilde{\mathcal{C}}_{\mu},
\end{aligned}
$$

where we denotes $\int_{q}=\int \frac{d^{4} q}{(2 \pi)^{3}} \bar{\epsilon}(q \cdot n)$. Moreover, performing similar computations as in the case for $\partial_{\mu} J^{\mu}$, the divergence of the energy-momentum gives rise to

$$
\begin{aligned}
\partial_{\mu} T^{\mu \nu}= & F^{\nu \mu} J_{\mu}+2 \int_{q} \delta\left(q^{2}\right) \\
& \times\left[q^{\nu} q^{\mu}+\frac{\hbar \epsilon^{\sigma \mu \alpha \beta}}{4}\left(\delta_{\sigma}^{\nu}\left(q_{\beta} \partial_{\alpha}+F_{\alpha \beta}\right)+q^{\nu} F_{\alpha \beta} \partial_{q \sigma}\right)\right] \tilde{\mathcal{C}}_{\mu} .
\end{aligned}
$$

The detailed derivation is shown in Appendix C. For collisions in practical systems obeying charge and energy-momentum conservation, the collisional terms in Eqs. (9) and (10) should automatically vanish. In contrast, in the RTA, the charge and energy-momentum conservations give matching conditions. In particular, when $\mathcal{A}_{\mu}=0$, we find that Eqs. (9) and (10) become

$$
\begin{aligned}
\partial_{\mu} J^{\mu} & =-\frac{\hbar}{4 \pi^{2}} E_{\mu} B^{\mu}-\frac{u_{\mu} \delta J^{\mu}}{\tau_{R}}, \\
\partial_{\mu} T^{\mu \nu} & =F^{\nu \mu} J_{\mu}-\frac{u_{\mu} \delta T^{\mu \nu}}{\tau_{R}}
\end{aligned}
$$

In this case, the matching conditions turns to the standard ones, $u_{\mu} \delta J^{\mu}=u_{\mu} \delta T^{\mu \nu}=0$. The vanishing nonequilibrium modifications upon charge density and energy-density current consequently allow us to consistently define the temperature and chemical potential in equilibrium as alternatively indicated in Ref. [53]. Taking other approximations could result in distinct conserved quantities without consistent physical interpretations.

\section{NONEQUILIBRIUM RESPONSES AND CHARGE CURRENTS}

Following Ref. [48], the perturbative solution for the nonequilibrium distribution function is given by

$$
\delta f_{q}=-\frac{\tau_{R}}{(q \cdot u)}\left(1-\hbar \frac{q^{\mu} \mathcal{A}_{\mu}}{(q \cdot u)}\right) \square f_{q}^{\mathrm{eq}},
$$

where $\mathcal{A}_{\mu}$ is taken to be a constant here. We may make the decomposition, $\delta f_{q}=\delta f_{q}^{(c)}+\delta f_{q}^{(Q)}$, where the superindices $(c)$ and $(Q)$ correspond to the classical and quantum corrections, respectively. We then further separate the part for ideal fluids, and the viscous correction, $\delta f^{(c / Q)}=$ $\delta_{I} f_{q}^{(c / Q)}+\delta_{v} f_{q}^{(c / Q)}$, where the subindices $I$ and $v$ denote the inviscid and viscous parts. The explicit expression of $\delta_{I} f_{q}$ can be found in Ref. [48]. In light of Ref. [48], we decompose the quantum corrections of the nonequilibrium distribution function into three parts as $\delta_{v} f_{q}^{(Q)}=$ $\delta_{v} f_{q}^{\mathcal{K}}+\delta_{v} f_{q}^{\mathcal{H}}+\delta_{v} f_{q}^{\mathcal{C}}$, where $\delta_{v} f_{q}^{\mathcal{K}}$ is led by the perturbative solution out of equilibrium solved from CKT and $\delta_{v} f_{q}^{\mathcal{H}}$ is attributed to the $\hbar$ corrections of the temporal derivatives $(u \cdot \partial)$ upon $T, \bar{\mu} \equiv \mu / T$, and $u^{\mu}$ from hydrodynamic EOM obtained from Eq. (8). Finally, $\delta_{v} f_{q}^{\mathcal{C}}$ comes from the $\hbar$ corrections in the collisional kernel, while this term depends on the assumption of $\mathcal{A}_{\mu}$ in the RT approximation, which does not play a significant role in our analysis. In Ref. [48] for an inviscid case, $\mathcal{A}_{\mu}$ is treated as a constant. We will follow the same convention in the computation of viscous corrections for consistency although $\mathcal{A}_{\mu}$ can be an operator governed by the frame transformation. On the other hand, as discussed previously, we may set $\mathcal{A}^{\mu}=0$ in the comoving frame for self-consistency to introduce local equilibrium thermodynamical parameters suggested by the matching conditions.

For convenience, hereafter we denote $q_{0} \equiv q \cdot u$ and $D \equiv u \cdot \partial$. By implementing CKT with the RT approximation, in the local rest frame, we find

$$
\delta_{v} f_{q}^{(c)}=-\frac{\tau_{R}}{q_{0}} q^{\mu} q^{\nu} \pi_{\mu \nu} \partial_{q_{0}} f_{q}^{(0)}
$$

with $f_{q}^{(0)}=1 /\left(e^{\left(q_{0}-\mu\right) / T}+1\right)$ and 


$$
\begin{aligned}
\delta_{v} f_{q}^{\mathcal{K}}= & \frac{\hbar \tau_{R}}{2 q_{0}}\left[\frac{B^{\mu}}{q_{0}}\left(\frac{2 q_{\mu} \theta}{3}-\pi_{\mu \nu} q^{\nu}\right)-2 \omega^{\mu} \pi_{\mu \nu} q^{\nu}\right. \\
& +\frac{(q \cdot \omega)}{q_{0}^{2}}\left(q^{\mu} q^{\nu} \pi_{\mu \nu}+\frac{q_{\perp}^{2}}{3} \theta\right)\left(1-q_{0} \partial_{q_{0}}\right)-\frac{2 q \cdot \omega}{3} \theta \\
& \left.-\frac{\epsilon^{\mu \nu \alpha \beta}}{q_{0}} u_{\mu} q_{\alpha} q^{\rho} \pi_{\nu \rho}\left(T \partial_{\beta} \bar{\mu}+\frac{q_{0} \partial_{\beta} T}{T}+q_{0} D u_{\beta}\right)\right] \partial_{q_{0}} f_{q}^{(0)},
\end{aligned}
$$

where we define $P^{\mu \nu} \equiv \eta^{\mu \nu}-u^{\mu} u^{\nu}$ as a projection operator with the Minkowski metric $\eta^{\mu \nu}=\operatorname{diag}(1,-1,-1,-1), \theta \equiv$ $\partial \cdot u$ as the bulk strength, $\pi^{\mu \nu} \equiv P_{\rho}^{\mu} P_{\sigma}^{\nu}\left(\partial^{\rho} u^{\sigma}+\partial^{\sigma} u^{\rho}-\right.$ $\left.2 \eta^{\rho \sigma} \theta / 3\right) / 2$ as the shear strength, and $V_{\perp}^{\mu} \equiv P_{\nu}^{\mu} V^{\nu}$ as the transverse component of an arbitrary vector $V^{\mu}$.

Next, we shall consider the viscous corrections on the hydrodynamic EOM. By solving Eq. (8) with the current and energy-momentum tensor in local equilibrium, it is found that $D T=-\theta / 3+\mathcal{O}(\hbar)$ and $D \bar{\mu}=\mathcal{O}(\hbar)$ with $\bar{\mu} \equiv \mu / T$, while the viscous correction does not lead to $\hbar$ corrections for $D T$ and $D \bar{\mu}$. We also include their contributions for the computation of currents. In addition, we find that the viscous correction gives rise to the $\hbar$ correction upon $D_{v} u_{\perp}^{\mu}$ and accordingly the hydrodynamic EOM results in

$$
\begin{aligned}
\delta_{v} f_{q}^{\mathcal{H}} & =-\hbar \tau_{R} D_{v} u_{\perp}^{\mu} \\
& =-\hbar \tau_{R} q_{\mu}\left[\tilde{U}_{\omega} T \omega_{\nu}\left(\frac{P^{\mu \nu} \theta}{6}-\pi^{\mu \nu}\right)+\tilde{U}_{B} B_{\nu} \pi^{\mu \nu}\right] \partial_{q_{0}} f_{q}^{(0)} .
\end{aligned}
$$

where $\quad \tilde{U}_{B}=-T^{2}\left(3 \bar{\mu}^{2}+\pi^{2}\right) /\left(48 p \pi^{2}\right) \quad$ and $\quad \tilde{U}_{\omega}=$ $T^{2} \bar{\mu}\left(\bar{\mu}^{2}+\pi^{2}\right) /\left(12 p \pi^{2}\right)$ with $p$ being pressure. On the other hand, the $\hbar$ corrections in collisions give

$$
\delta_{v} f_{q}^{\mathcal{C}}=\frac{\hbar \tau_{R}}{q_{0}^{4}}\left(q^{\mu} q^{\nu} \pi_{\mu \nu}+\frac{q_{\perp}^{2}}{3} \theta\right)(q \cdot \mathcal{A}) \partial_{q_{0}} f_{q}^{(0)} .
$$

From Eqs. (1) and (3), the quantum corrections of the nonequilibrium current reads

$$
\begin{aligned}
\delta J_{Q}^{\mu}= & 2 \hbar \int \frac{d^{4} q}{(2 \pi)^{3}} \bar{\epsilon}(q \cdot u) \delta\left(q^{2}\right) \\
& \times\left[q^{\mu} \delta f_{q}^{(Q)}+\left(S_{(u)}^{\mu \nu} \Delta_{\nu}-\frac{\epsilon^{\mu \nu \alpha \beta}}{4} F_{\alpha \beta} \partial_{q \nu}\right) \delta f_{q}^{(c)}\right] .
\end{aligned}
$$

By inserting $\delta f_{q}^{(Q)}$ and $\delta f_{q}^{(c)}$ into Eq. (17), we obtain the quantum correction upon the charge current, $\delta J_{Q \perp}^{\mu}=$ $\delta J_{I Q \perp}^{\mu}+\delta J_{v Q \perp}^{\mu}$, where $\delta J_{I Q \perp}^{\mu}$ as the part for ideal fluids is shown in Ref. [48]. The viscous part takes the form

$$
\delta_{v} J_{Q \perp}^{\mu}=\hbar\left(\delta \sigma_{B}^{\mu \nu} B_{\nu}+\delta \sigma_{\omega}^{\mu \nu} \omega_{\nu}+\delta \sigma_{\mathcal{A}}^{\mu \nu} \mathcal{A}_{\nu}\right) .
$$

When not applying the hydrodynamic EOM, only $\delta_{v} f_{q}^{\mathcal{K}}$ contributes and one finds

$$
\begin{aligned}
& \delta \sigma_{B}^{\mu \nu}=\frac{\tau_{R} \mu}{4 \pi^{2}}\left(\frac{4}{9} \theta P^{\mu \nu}-\pi^{\mu \nu}\right), \\
& \delta \sigma_{\omega}^{\mu \nu}=-\frac{\tau_{R} I_{1} T^{2}}{36 \pi^{2}}\left(\frac{17 P^{\mu \nu} \theta}{3}+20 \pi^{\mu \nu}\right),
\end{aligned}
$$

and

$$
\delta \sigma_{\mathcal{A}}^{\mu \nu}=-\frac{\tau_{R} \mu}{18 \pi^{2}}\left(\frac{5 P^{\mu \nu} \theta}{3}+2 \pi^{\mu \nu}\right)
$$

where $I_{1}=\bar{\mu}^{2}+\pi^{2} / 3$. The results suggest that the viscous corrections upon CME and CVE conductivities should exist even for an open system in which the backreaction on environments is neglected and the energy-momentum conservation is violated such as the case in Weyl semimetals when the scattering between quasiparticles and impurities dominate the interactions among quasiparticles.

Now, for the right-handed chiral fluid as a closed systems with energy-momentum conservation, by implementing the hydrodynamic EOM, the coefficients become

$$
\begin{aligned}
\delta \sigma_{B}^{\mu \nu}= & \frac{\tau_{R}}{12 \pi^{2}}\left[\frac{10}{3} \mu \theta P^{\mu \nu}-\left(3 \mu+2 I_{2} T^{3} \tilde{U}_{B}\right) \pi^{\mu \nu}\right], \\
\delta \sigma_{\omega}^{\mu \nu}= & -\frac{\tau_{R}}{12 \pi^{2}}\left[\frac{P^{\mu \nu} T^{2} \theta}{3}\left(I_{1}+I_{2} T^{2} \tilde{U}_{\omega}\right)\right. \\
& \left.+\pi^{\mu \nu} T^{2}\left(\frac{20}{3} I_{1}-2 I_{2} T^{2} \tilde{U}_{\omega}\right)\right],
\end{aligned}
$$

and

$$
\delta \sigma_{\mathcal{A}}^{\mu \nu}=-\frac{\tau_{R} \mu}{9 \pi^{2}} \pi^{\mu \nu},
$$

where $I_{2}=\bar{\mu}\left(\bar{\mu}^{2}+\pi^{2}\right)$. Note that there exist two terms in $\delta f_{q}^{I(Q)}, \quad-\hbar \tau_{R}(2 T)^{-1}(q \cdot \omega)(D T)\left(1-q_{0} \partial_{q_{0}}\right) f_{q}^{(0)}$ and $-\hbar \tau_{R}\left(2 q_{0}\right)^{-2}(q \cdot \partial)(q \cdot \omega)$, which also contribute to viscous corrections with vorticity when the hydrodynamic EOM are applied in computations. In general, by redefining $u^{\mu}$, one can shift the above corrections on CME/CVE conductivities to the transport coefficients of energy density currents. It is more enlightening to simplify the expressions of $\delta \sigma_{B}^{\mu \nu}$ and $\delta \sigma_{\omega}^{\mu \nu}$ in distinct limits. In the high-temperature limit $(\bar{\mu} \ll 1)$, the coefficients in Eq. (18) reduce to

$$
\begin{aligned}
& \delta \sigma_{B}^{\mu \nu}=\hbar \tau_{R} \mu\left(\frac{5 P^{\mu \nu}}{18 \pi^{2}} \theta-\frac{\pi^{\mu \nu}}{14 \pi^{2}}\right), \\
& \delta \sigma_{\omega}^{\mu \nu}=-\hbar \tau_{R} T^{2}\left[\frac{P^{\mu \nu}}{108} \theta\left(1+\frac{111 \bar{\mu}^{2}}{7 \pi^{2}}\right)-\frac{5 \pi^{\mu \nu}}{27}\left(1-\frac{6 \bar{\mu}^{2}}{7 \pi^{2}}\right)\right] .
\end{aligned}
$$


On the contrary, in the low-temperature limit $(\bar{\mu} \gg 1)$, one obtains

$$
\begin{aligned}
& \delta \sigma_{B}^{\mu \nu}=\hbar \tau_{R} \mu\left(\frac{5 P^{\mu \nu}}{18 \pi^{2}} \theta-\frac{\pi^{\mu \nu}}{6 \bar{\mu}^{2}}\right), \\
& \delta \sigma_{\omega}^{\mu \nu}=-\hbar \tau_{R} \mu^{2}\left(\frac{P^{\mu \nu}}{12 \pi^{2}} \theta-\frac{2 \pi^{\mu \nu}}{9 \pi^{2}}\right) .
\end{aligned}
$$

Despite the complexity of computations, it is worthwhile to note that such viscous corrections actually originate from Bianchi identities $\partial_{\nu} \tilde{F}^{\mu \nu}=\partial_{\nu} \tilde{\omega}^{\mu \nu}=0$, where $\tilde{\omega}^{\mu \nu}=$ $\frac{1}{2} \epsilon^{\mu \nu \alpha \beta} \partial_{\alpha} u_{\beta}$, which relate the temporal derivatives of $B^{\mu}$ and $\omega^{\mu}$ (in the fluid comoving frame) to their couplings with the gradients of $u^{\mu}$ as shown in Ref. [48]. Such an origin is a reminiscence of the $\mathrm{AC}$ conductivity of $\mathrm{CME}$ driven by time-dependent $B^{\mu}$ and the viscous corrections upon CME/CVE are expected to be dissipative.

So far, we have only considered the contributions for right-handed fermions. The quantum corrections for lefthanded fermions will yield the same results but with the change of an overall sign for each term at $\mathcal{O}(\hbar)$. Since $P^{\mu \nu} \theta$ and $\pi^{\mu \nu}$ are even under the parity $(\mathcal{P})$ transformation, it is anticipated that the transport coefficients of the nonequilibrium corrections on CME/CVE have the same parity as those in equilibrium, which can be more apparently observed from the simplified expressions shown in two limits above. Therefore, the bulk and shear strengths not only affect the vector currents $\left(J_{V}^{\mu}=J_{R}^{\mu}+J_{L}^{\mu}\right)$ induced by CME/CVE with nonzero axial-charge chemical potentials $\left(\mu_{A}=\mu_{R}-\mu_{L}\right)$ but also the axial currents $\left(J_{A}^{\mu}=J_{R}^{\mu}-J_{L}^{\mu}\right)$ from the chiral separation effect(CSE) [59] and CVE with nonzero vectorcharge chemical potentials $\left(\mu_{V}=\mu_{R}+\mu_{L}\right)$. It is worthwhile to note that such second-order quantum corrections on currents have different symmetry properties compared to the second-order classical effects also pertinent to magnetic fields such as the Hall-diffusion currents $J_{\perp}^{\mu} \sim$ $\tau_{R}^{2} \epsilon^{\mu \nu \alpha \beta} u_{\nu} B_{\alpha} \partial_{\beta} \mu^{2}$ in Ref. [51]. Because there is no sign flipping for right/left-handed fermions in the classical case, the corresponding axial current can only be generated when $\mu_{A} \neq 0\left(\right.$ or $\left.\partial_{\perp \beta} \mu_{A} \neq 0\right)$, which is analogous to CESE $[44,45]$.

\section{ENTROPY PRODUCTION}

In contrast to the anomalous transport in equilibrium, the nonequilibrium quantum corrections of the charge current $\delta J_{Q}^{\mu}$ are dissipative. This is foreseen by the timereversal symmetry $(\mathcal{T})$. Since the charge current $J_{\perp}^{\mu}, B^{\mu}$, and $\omega^{\mu}$ are $\mathcal{T}$-odd and $E^{\mu}$ is $\mathcal{T}$-even, from the classical ohmic current led by the RT approximation $J_{\perp}^{\mu} \propto \tau_{R} E^{\mu}$, one finds that $\tau_{R}$ is also $\mathcal{T}$-odd, whereas the $\mathrm{CME} / \mathrm{CVE}$ conductivities in equilibrium are $\mathcal{T}$-even and thus nondissipative. Nonetheless, because $\theta P^{\mu \nu}$ and $\pi^{\mu \nu}$ are both $\mathcal{T}$-odd, the corresponding transport coefficients of the viscous corrections upon chiral magnetic/vortical currents are proportional to $\tau_{R}$ multiplied by $\mathcal{T}$-even functions of $T$ and $\mu$, which accordingly yield dissipation. The same arguments can be applied to the nonequilibrium transport found in inviscid cases [48]. Notably, in light of symmetry, one finds $\mathcal{A}^{\mu} \sim B^{\mu}$ or $\mathcal{A}^{\mu} \sim \omega^{\mu}$ for viscous corrections. Consequently, the $\hbar$ corrections in practical collisions can only affect the prefactors of transport coefficients without altering their structures. Overall, the nonequilibrium second-order quantum transport is parity-odd and dissipative as opposed to the classical one (e.g., classical Hall effects), which is parity even and nondissipative.

Albeit the nonequilibrium quantum transport results in dissipation, its entropy production is suppressed by the dissipation from classical effects as shown below. We may introduce the entropy density current in an usual form as for relativistic hydrodynamics,

$s^{\mu}=\frac{1}{T}\left(p u^{\mu}+T^{\mu \nu} u_{\nu}-\mu J^{\mu}\right)+\hbar\left(D_{B} B^{\mu}+D_{\omega} \omega^{\mu}\right)$,

where the nondissipative corrections proportional to $B^{\mu}$ and $\omega^{\mu}$ originating from the nondissipative charge and energydensity currents. The coefficients $D_{B}$ and $D_{\omega}$ should be determined by the transport coefficients of CME and CVE in equilibrium. See e.g., Ref. [18] for details. The explicit form of $D_{B(\omega)}$ is not important in our discussion.

The constitutive relation now can be written as $T^{\mu \nu}=$ $u^{\mu} u^{\nu} \epsilon-p P^{\mu \nu}+\Pi_{\mathrm{dis}}^{\mu \nu}+\Pi_{\mathrm{non}}^{\mu \nu}$, where $\Pi_{\mathrm{dis}}^{\mu \nu}$ denotes the dissipative corrections characterized by viscous effects and $\Pi_{\text {non }}^{\mu \nu}$ corresponds to the nondissipative corrections led by anomalous effects in equilibrium. When $\mathcal{A}_{\mu}=0$, based on the matching conditions such that $u_{\mu} \delta J^{\mu}=u_{\mu} \delta T^{\mu \nu}=0$, we find

$$
\partial_{\mu} s^{\mu}=\frac{1}{T}\left[\Pi_{\mathrm{dis}}^{\mu \nu} \partial_{\mu} u_{\nu}-\left(E_{\mu}+T \partial_{\mu} \bar{\mu}\right) \delta J^{\mu}\right] .
$$

In Eq. (24), the classical contributions result in positive entropy production at $\mathcal{O}\left(\partial^{2}\right)$, while the corresponding quantum corrections are at $\mathcal{O}\left(\hbar \partial^{3}\right)$. Although the nonequilibrium quantum corrections here could be either positive or negative, they are always suppressed by the classical contributions and the second law of thermodynamics is satisfied.

\section{DISCUSSIONS AND OUTLOOK}

Regarding the validity of our findings, due to the gradient expansion, the results should be legitimate for $\tau_{R} \partial \ll 1$, which imparts an upper bound for $\tau_{R}$. In addition, given that the CKT itself is subject to weakly coupled systems, $\tau_{R}$ cannot be too small. More generally, although the CKT is developed to cope with nonequilibrium conditions, it is applicable for small gradients and weak background fields based on the $\hbar$ expansion. To extend the validity beyond such limits, we have to solve for Wigner functions nonperturbatively from Kadanoff-Baym 
equations (derived from Schwinger-Dyson equations), which could be a formidable analytic problem.

Nonetheless, we can still perturbatively solve for higherorder corrections in the $\hbar$ expansion (see Ref. [60] for a relevant study). Alternatively, one may also construct Wigner functions for nonequilibrium situations directly from Landau-level wave functions involving all-order $\hbar$ corrections, while it is subject to the case with just constant magnetic fields. Both approaches are systematic derivations of CKT from quantum field theory, which also complement each other. Up to $\mathcal{O}\left(\hbar^{2}\right)$, it is anticipated that novel nondissipative quantum effects such as the charge modification led by $\omega \cdot B$ discovered in Ref. [61] will be found. Furthermore, for more general cases, one may include dynamical gauge fields, which could yield chiral-plasma instabilities [62] and more profound phenomena. Such instabilities can be treated in our hydrodynamics approach if the time and length scales of the instabilities are much larger than the mean free time and length. The typical time and length scales of the instabilities are evaluated as $\tau_{\text {inst }} \sim$ $\tau_{R} m_{D}^{2} \ell_{\text {inst }}^{2}$ and $\ell_{\text {inst }} \sim 1 /\left(\alpha \mu_{A}\right)$, where $\alpha=e^{2} /(4 \pi)$ and $m_{D}$ is the Debye mass, while the mean free time and path are of order $\tau_{R}$ [62]. Therefore, $\tau_{\text {inst }} \gg \tau_{R}$ and $\ell_{\text {inst }} \gg \tau_{R}$ lead to conditions $m_{D}^{2} \gg\left(\alpha \mu_{A}\right)^{2}$ and $\tau_{R} \ll 1 /\left(\alpha \mu_{A}\right)$. The former is satisfied at high temperature $T \gg \sqrt{\alpha} \mu_{A}$ or density $\mu_{V} \gg \sqrt{\alpha} \mu_{A}$, where $m_{D}^{2} \sim \alpha T^{2}$ or $\alpha \mu_{V}^{2}$. The latter depends on the strength of interaction. For example, if we evaluate $\tau_{R}$ in a quark gluon plasma, it behaves $\tau_{R} \sim \alpha_{s} T \ln \alpha_{s}^{-1}$, where $\alpha_{s}$ is the strong coupling constant; thus, the condition reduces to $T \gg \alpha \mu_{A} /\left(\alpha_{s} \ln \alpha_{s}^{-1}\right)$. If the opposite limit is realized, we have to employ the chiral kinetic equation to describe the dynamics of chiral kinetic instabilities.

\section{ACKNOWLEDGMENTS}

The authors thank I. Shovkovy for useful discussions. Y.H. was partially supported by Japan Society of Promotion of Science (JSPS), Grants-in-Aid for Scientific Research (KAKENHI) Grants No. 15H03652, No. 16K17716, and No. 17H06462. Y. H. was also partially supported by RIKEN iTHES Project and iTHEMS Program. D. Y. was supported by the RIKEN Foreign Postdoctoral Researcher program.

\section{APPENDIX A: WIGNER FUNCTIONS WITH QUANTUM CORRECTIONS}

Based on the Dirac equations under the Wigner transformation up to $\mathcal{O}(\hbar)$, we shall obtain the following Kaddanof-Baym-like equations for right-handed fermions,

$$
\begin{aligned}
\sigma^{\mu}\left(q_{\mu}+\frac{i \hbar}{2} \Delta_{\mu}\right) \dot{S}^{<} & =\frac{i \hbar}{2}\left(\Sigma^{<} \dot{S}^{>}-\Sigma^{>} \grave{S}^{<}\right), \\
\left(q_{\mu}-\frac{i \hbar}{2} \Delta_{\mu}\right) \grave{S}^{<} \sigma^{\mu} & =-\frac{i \hbar}{2}\left(\grave{S}^{>} \Sigma^{<}-\grave{S}^{<} \Sigma^{>}\right),
\end{aligned}
$$

By parametrizing $\grave{S}^{<}=\bar{\sigma}^{\mu} \grave{S}_{\mu}^{<}$, the above equation yield the difference equations,

$$
\begin{aligned}
\hbar\left\{\sigma^{\mu}, \bar{\sigma}^{\nu}\right\} \mathcal{D}_{\mu} \grave{S}_{\nu}^{<} & =2 i\left[\sigma^{\mu}, \bar{\sigma}^{\nu}\right] q_{\mu} \grave{S}_{\nu}^{<}, \\
\hbar\left[\sigma^{\mu}, \bar{\sigma}^{\nu}\right] \mathcal{D}_{\mu} \grave{S}_{\nu}^{<} & =2 i\left\{\sigma^{\mu}, \bar{\sigma}^{\nu}\right\} q_{\mu} \grave{S}_{\nu}^{<},
\end{aligned}
$$

where $[A, B]=A B-B A$ and $\{A, B\}=A B+B A$ and

$$
\mathcal{D}_{\mu} \grave{S}_{\nu}^{<}=\Delta_{\mu} \grave{S}_{\nu}^{<}-\Sigma_{\mu}^{<} \grave{S}_{\nu}^{>}+\Sigma_{\mu}^{>} \grave{S}_{\nu}^{<}
$$

with $\Delta_{\mu}=\partial_{\mu}+F_{\nu \mu} \frac{\partial}{\partial q_{\nu}}$. We should now perturbatively solve for $\grave{S}_{\mu}^{<}$from Eq. (A3) up to $\mathcal{O}(\hbar)$. We thus make the ansatz, $\grave{S}_{\mu}^{<}=\grave{S}_{\mu}^{<(0)}+\hbar \delta \grave{S}_{\mu}^{<}$. It is easy to find that

$$
\grave{S}_{\mu}^{<(0)}=2 \pi \delta\left(q^{2}\right) q_{\mu} f(q, X)
$$

which is independent of a choice for the basis of spins. We may now define $n \cdot \sigma=I$ by introducing a frame vector $n^{\mu}$ normalized as $n^{2}=1$ and its corresponding projection operator $P^{\mu \nu}=\eta^{\mu \nu}-n^{\mu} n^{\nu}$ giving $P^{\mu \nu} n_{\nu}=0$. The difference equations then become

$$
\mathcal{D} \cdot \grave{S}^{<}=0, \quad q \cdot \grave{S}^{<}=0
$$

and up to $\mathcal{O}(\hbar)$,

$$
\begin{aligned}
2 \pi \delta\left(q^{2}\right) \sigma_{\perp}^{\mu}\left(q \cdot n \mathcal{D}_{\mu}-q_{\mu} n \cdot \mathcal{D}\right) f & =-2 \sigma_{\perp}^{\mu} \epsilon_{\alpha \mu \nu \beta} n^{\alpha} q^{\nu} \delta \grave{S}^{<\beta}, \\
2 \pi \sigma_{\perp}^{\mu} \epsilon_{\alpha \mu \nu \beta} \delta\left(q^{2}\right) n^{\alpha} q^{\nu} \mathcal{D}^{\beta} f & =2 \sigma_{\perp}^{\mu}\left(q \cdot n \delta \grave{S}_{\mu}^{<}-q_{\mu} n \cdot \delta \grave{S}^{<}\right),
\end{aligned}
$$

where $\sigma_{\perp}^{\mu}=P^{\mu \nu} \sigma_{\nu}$ and $\mathcal{D}_{\beta} f=\Delta_{\beta} f-\mathcal{C}_{\beta}$ and $\mathcal{C}_{\beta}=\Sigma_{\beta}^{<} \bar{f}-\Sigma_{\beta}^{>} f$. When taking $n^{\mu}=(1, \mathbf{0})$, Eq. (A7) reduces to Eq. (33) in Ref. [36]. Solving Eq. (A7), one derive

$$
\begin{aligned}
\grave{S}^{<\mu}(q, X)= & 2 \pi\left(q^{\mu} \delta\left(q^{2}\right)+\hbar \delta\left(q^{2}\right) S_{(n)}^{\mu \nu} \mathcal{D}_{\nu}\right. \\
& \left.+\hbar \epsilon^{\mu \nu \alpha \beta} q_{\nu} F_{\alpha \beta} \frac{\partial \delta\left(q^{2}\right)}{2 \partial q^{2}}\right) f(q, X),
\end{aligned}
$$

where

$$
S_{(n)}^{\mu \nu}=\frac{\epsilon^{\mu \nu \alpha \beta}}{2(q \cdot n)} q_{\alpha} n_{\beta}
$$




\section{APPENDIX B: FRAME TRANSFORMATION FOR THE RTA}

To preserve Lorentz invariance of the CKT in Eq. (4) with the RTA, we have to introduce the frame transformation on $\mathcal{A}_{\mu}$. By implementing Eqs. (4) and (7) can be rewritten as

$$
\delta\left(q^{2}\right)\left(\square^{(n)} f_{q}^{(n)}+\frac{\hbar B^{(n) \mu}}{2 q \cdot n} \partial_{q \mu}\left(q \cdot \Delta f_{q}^{(n)}\right)\right)=I_{c}^{(n)},
$$

with

$$
\begin{aligned}
I_{c}^{(n)}= & -\frac{\delta\left(q^{2}\right)}{\tau_{R}}\left(\left(q \cdot u+\frac{\hbar q \cdot \mathcal{A}^{(n)}}{(q \cdot u)^{2}}\right) \delta f_{q}^{(n)}\right. \\
& \left.+\frac{\hbar B^{(n) \mu}}{2 q \cdot n} \partial_{q \mu}\left(q \cdot u \delta f_{q}^{(n)}\right)\right),
\end{aligned}
$$

where we use the superindices ${ }^{(n)}$ to track the framedependent terms explicitly. Also, we have applied the relations,

$$
\delta\left(q^{2}-\hbar \frac{B^{(n)} \cdot q}{q \cdot n}\right)=\delta\left(q^{2}\right)-\frac{\hbar B^{(n) \mu}}{2 q \cdot n} \partial_{q \mu} \delta\left(q^{2}\right),
$$

and

$\left(\partial_{q \mu} \delta\left(q^{2}\right)\right) q \cdot \mathcal{D} f_{q}^{(n)}=-\delta\left(q^{2}\right) \partial_{q \mu}\left(q \cdot \mathcal{D} f_{q}^{(n)}\right)+\mathcal{O}(\hbar)$.

Recall that the full CKT in Eq. (B1) is frame independent (Lorentz invariant) when considering also the nontrivial frame transformation upon the distribution functions. According to Ref. [36], the frame transformation for the distribution function reads

$$
f_{q}^{\left(n^{\prime}\right)}=f_{q}^{(n)}+\hbar N_{n n^{\prime}}^{\nu} \mathcal{D}_{\nu} f_{q}^{(n)},
$$

where

$$
N_{n n^{\prime}}^{\nu}=\frac{\epsilon^{\mu \nu \alpha \beta} q_{\alpha} n_{\beta} n_{\mu}^{\prime}}{2(q \cdot n)\left(q \cdot n^{\prime}\right)} .
$$

When taking $n^{\mu}=u^{\mu}$ and using the RTA, one finds that Eq. (B4) reduces to

$$
f_{q}^{\left(n^{\prime}\right)}=f_{q}^{(u)}+\hbar N_{u n^{\prime}}^{\nu} \Delta_{\nu} f_{q}^{(u)},
$$

which is independent of collisions. Note that the equation above also works for $\delta f_{q}^{\left(n^{\prime}\right)}$. Consequently, the collisional part alone in Eq. (B1) should be frame independent. By utilizing $I_{c}^{\left(n^{\prime}\right)}-I_{c}^{(u)}=0$ and $\delta f_{q}^{\left(n^{\prime}\right)}=\delta f_{q}^{(u)}+\hbar N_{u n^{\prime}}^{\nu} \Delta_{\nu} \delta f_{q}^{(u)}$, we obtain

$$
\begin{aligned}
\mathcal{A}_{\nu}^{\left(n^{\prime}\right)}= & \mathcal{A}_{\nu}^{(u)}-u_{\nu}(q \cdot u)^{2}\left(N_{u n^{\prime}}^{\alpha} \Delta_{\alpha}+\frac{1}{2(q \cdot u)}\left(\frac{B^{\left(n^{\prime}\right) \alpha}}{q \cdot n^{\prime}}-\frac{B^{(u) \alpha}}{q \cdot u}\right)\right. \\
& \left.\times\left(u_{\alpha}+(q \cdot u) \partial_{q \alpha}\right)\right)
\end{aligned}
$$

which gives the frame transformation upon the collisional terms in a RTA.

\section{APPENDIX C: DIVERGENCE OF THE ENERGY-MOMENTUM TENSOR}

Here we present some critical steps for the derivation of Eq. (10). Following the trick in Ref. [53], we find

$$
\begin{aligned}
\partial_{\mu} T^{\mu \nu} & =\int \frac{d^{4} q}{(2 \pi)^{4}}\left(2 q^{\nu} \partial \cdot S^{<}+q \cdot \partial S^{<\nu}-q^{\nu} \partial \cdot S^{<}\right) \\
& =\int \frac{d^{4} q}{(2 \pi)^{4}}\left(2 q^{\nu} \partial \cdot S^{<}-\frac{1}{2} \epsilon^{\nu \kappa \sigma \rho} \epsilon_{\mu \lambda \sigma \rho} q^{\lambda} \partial_{\kappa} S^{<\mu}\right) .
\end{aligned}
$$

Performing straightforward computations, one should obtain

$$
\begin{aligned}
\epsilon_{\mu \lambda \sigma \rho} q^{\lambda} \partial_{\kappa} S^{<\mu}= & -\hbar \pi \bar{\epsilon}(q \cdot n)\left[2 \delta\left(q^{2}\right) q_{\sigma} \partial_{\kappa}\left(F_{\rho \lambda} \partial_{q}^{\lambda} f_{q}+\mathcal{C}_{\rho}\right)\right. \\
& +F_{\sigma \rho} q^{\lambda}\left(\partial_{q \lambda} \delta\left(q^{2}\right)\right)\left(\partial_{\kappa} f_{q}\right) \\
& \left.+2 q_{\sigma}\left(\partial_{q}^{\lambda} \delta\left(q^{2}\right)\right) \partial_{\kappa}\left(F_{\rho \lambda} f_{q}\right)\right],
\end{aligned}
$$

which results in

$$
\begin{aligned}
& \int \frac{d^{4} q}{(2 \pi)^{4}}\left(q \cdot \partial S^{<\nu}-q^{\nu} \partial \cdot S^{<}\right) \\
&= \hbar \int_{q} \delta\left(q^{2}\right) \frac{\epsilon^{\nu \kappa \sigma \rho}}{2}\left[F_{\rho \sigma}\left(1+\frac{q^{\gamma} \partial_{q \gamma}}{2}\right) \partial_{\kappa} f_{q}+q_{\sigma} \partial_{\kappa} \mathcal{C}_{\rho}\right] \\
& \quad=\hbar \int_{q} \delta\left(q^{2}\right) \frac{\epsilon^{\nu \kappa \sigma \rho}}{2} q_{\sigma} \partial_{\kappa} \mathcal{C}_{\rho},
\end{aligned}
$$

where the first term on the right-hand side of the first equality in fact vanishes. On the other hand, we find

$$
\begin{aligned}
& \int \frac{d^{4} q}{(2 \pi)^{4}} 2 q^{\nu} \partial \cdot S^{<} \\
& \quad=-2 \int \frac{d^{4} q}{(2 \pi)^{4}} q^{\nu}\left(F_{\rho \mu} \partial_{q}^{\rho} S^{<\mu}-\Sigma^{<} \cdot S^{>}+\Sigma^{>} \cdot S^{<}\right) .
\end{aligned}
$$

By performing the integration by part and dropping the divergent and vanishing surface terms, we obtain

$$
\begin{aligned}
& \int \frac{d^{4} q}{(2 \pi)^{4}} 2 q^{\nu} \partial \cdot S^{<} \\
& =2 \int \frac{d^{4} q}{(2 \pi)^{4}}\left[F^{\nu \mu} S_{\mu}^{<}+2 \pi \bar{\epsilon}(q \cdot n) \delta\left(q^{2}\right)\right. \\
& \left.\quad \times\left(q^{\nu} q^{\mu}-\frac{\hbar}{4} q^{\nu} \epsilon^{\mu \sigma \alpha \beta} F_{\alpha \beta} \partial_{q \sigma}-\frac{\hbar}{4} \epsilon^{\mu \nu \alpha \beta} F_{\alpha \beta}\right)\right] \tilde{\mathcal{C}}_{\mu},
\end{aligned}
$$

up to $\mathcal{O}(\hbar)$. Combining Eqs. (C3) and (C5), we acquire Eq. (10). 
[1] A. Vilenkin, Phys. Rev. D 20, 1807 (1979).

[2] D. E. Kharzeev, L. D. McLerran, and H. J. Warringa, Nucl. Phys. A803, 227 (2008).

[3] K. Fukushima, D. E. Kharzeev, and H. J. Warringa, Phys. Rev. D 78, 074033 (2008).

[4] Q. Li, D. E. Kharzeev, C. Zhang, Y. Huang, I. Pletikosic, A. V. Fedorov, R. D. Zhong, J. A. Schneeloch, G. D. Gu, and T. Valla, Nat. Phys. 12, 550 (2016).

[5] D. E. Kharzeev and H. J. Warringa, Phys. Rev. D 80, 034028 (2009).

[6] K. Landsteiner, E. Megias, and F. Pena-Benitez, Phys. Rev. Lett. 107, 021601 (2011).

[7] J.-H. Gao, Z.-T. Liang, S. Pu, Q. Wang, and X.-N. Wang, Phys. Rev. Lett. 109, 232301 (2012).

[8] D. T. Son and N. Yamamoto, Phys. Rev. Lett. 109, 181602 (2012).

[9] M. Stephanov and Y. Yin, Phys. Rev. Lett. 109, 162001 (2012).

[10] D. T. Son and N. Yamamoto, Phys. Rev. D 87, 085016 (2013).

[11] J.-W. Chen, S. Pu, Q. Wang, and X.-N. Wang, Phys. Rev. Lett. 110, 262301 (2013).

[12] C. Manuel and J. M. Torres-Rincon, Phys. Rev. D 89, 096002 (2014).

[13] C. Manuel and J. M. Torres-Rincon, Phys. Rev. D 90, 076007 (2014).

[14] D. E. Kharzeev, M. A. Stephanov, and H.-U. Yee, Phys. Rev. D 95, 051901 (2017).

[15] A. Huang, Y. Jiang, S. Shi, J. Liao, and P. Zhuang, Phys. Lett. B 777, 177 (2018).

[16] J.-h. Gao, S. Pu, and Q. Wang, Phys. Rev. D 96, 016002 (2017).

[17] S. Ebihara, K. Fukushima, and S. Pu, Phys. Rev. D 96, 016016 (2017).

[18] D. T. Son and P. Surowka, Phys. Rev. Lett. 103, 191601 (2009).

[19] Y. Neiman and Y. Oz, J. High Energy Phys. 03 (2011) 023.

[20] A. V. Sadofyev and M. V. Isachenkov, Phys. Lett. B 697, 404 (2011).

[21] S. Pu, J.-h. Gao, and Q. Wang, Phys. Rev. D 83, 094017 (2011).

[22] D. E. Kharzeev and H.-U. Yee, Phys. Rev. D 84, 045025 (2011).

[23] M. Abramczyk, T. Blum, G. Petropoulos, and R. Zhou, Proc. Sci., LAT2009 (2009) 181 [arXiv:0911.1348].

[24] P. Buividovich, M. Chernodub, E. Luschevskaya, and M. Polikarpov, Phys. Rev. D 80, 054503 (2009).

[25] P. Buividovich, E. Luschevskaya, M. Polikarpov, and M. Chernodub, JETP Lett. 90, 412 (2009).

[26] P. Buividovich, M. Chernodub, D. Kharzeev, T. Kalaydzhyan, E. Luschevskaya, and M. I. Polikarpov, Phys. Rev. Lett. 105, 132001 (2010).

[27] A. Yamamoto, Phys. Rev. Lett. 107, 031601 (2011).

[28] N. Müller, S. Schlichting, and S. Sharma, Phys. Rev. Lett. 117, 142301 (2016).

[29] M. Mace, N. Mueller, S. Schlichting, and S. Sharma, Phys. Rev. D 95, 036023 (2017).

[30] J. Erdmenger, M. Haack, M. Kaminski, and A. Yarom, J. High Energy Phys. 01 (2009) 055.
[31] M. Torabian and H.-U. Yee, J. High Energy Phys. 08 (2009) 020.

[32] N. Banerjee, J. Bhattacharya, S. Bhattacharyya, S. Dutta, R. Loganayagam, and P. Surówka, J. High Energy Phys. 01 (2011) 094.

[33] K. Landsteiner, E. Megias, L. Melgar, and F. Pena-Benitez, J. High Energy Phys. 09 (2011) 121.

[34] J.-Y. Chen, D. T. Son, M. A. Stephanov, H.-U. Yee, and Y. Yin, Phys. Rev. Lett. 113, 182302 (2014).

[35] J.-Y. Chen, D. T. Son, and M. A. Stephanov, Phys. Rev. Lett. 115, 021601 (2015).

[36] Y. Hidaka, S. Pu, and D.-L. Yang, Phys. Rev. D 95, 091901 (2017).

[37] K. Jensen, R. Loganayagam, and A. Yarom, J. High Energy Phys. 02 (2013) 088.

[38] D.-F. Hou, H. Liu, and H.-c. Ren, Phys. Rev. D 86, 121703 (2012).

[39] S. Golkar and D. T. Son, J. High Energy Phys. 02 (2015) 169.

[40] K. Jensen, P. Kovtun, and A. Ritz, J. High Energy Phys. 10 (2013) 186.

[41] S. D. Chowdhury and J. R. David, J. High Energy Phys. 11 (2015) 048.

[42] S. Golkar and S. Sethi, J. High Energy Phys. 05 (2016) 105.

[43] S. D. Chowdhury and J. R. David, J. High Energy Phys. 12 (2016) 116.

[44] X.-G. Huang and J. Liao, Phys. Rev. Lett. 110, 232302 (2013).

[45] S. Pu, S.-Y. Wu, and D.-L. Yang, Phys. Rev. D 89, 085024 (2014).

[46] S. Pu, S.-Y. Wu, and D.-L. Yang, Phys. Rev. D 91, 025011 (2015).

[47] D. Satow and H.-U. Yee, Phys. Rev. D 90, 014027 (2014).

[48] Y. Hidaka, S. Pu, and D.-L. Yang, Phys. Rev. D 97, 016004 (2018).

[49] See Refs. [50,51] for related studies in open systems, where the energy-momentum conservation led by hydrodynamics is not considered.

[50] J.-W. Chen, T. Ishii, S. Pu, and N. Yamamoto, Phys. Rev. D 93, 125023 (2016).

[51] E. V. Gorbar, I. A. Shovkovy, S. Vilchinskii, I. Rudenok, A. Boyarsky, and O. Ruchayskiy, Phys. Rev. D 93, 105028 (2016).

[52] The viscous effects in the absence of background fields are incorporated in the study presented in Ref. [53], while the authors focus on the hydrodynamic dispersion relation modified by vorticity and the charge current is not computed therein.

[53] E. V. Gorbar, D. O. Rybalka, and I. A. Shovkovy, Phys. Rev. D 95, 096010 (2017).

[54] J. Crossno, J. K. Shi, K. Wang, X. Liu, A. Harzheim, A. Lucas, S. Sachdev, P. Kim, T. Taniguchi, K. Watanabe, T. A. Ohki, and K. C. Fong, Science 351, 1058 (2016).

[55] F. Ghahari, H.-Y. Xie, T. Taniguchi, K. Watanabe, M. S. Foster, and P. Kim, Phys. Rev. Lett. 116, 136802 (2016).

[56] A. Lucas, R. A. Davison, and S. Sachdev, Proc. Nat. Acad. Sci. 113, 9463 (2016). 
[57] E. V. Gorbar, V. A. Miransky, I. A. Shovkovy, and P. O. Sukhachov, Phys. Rev. B 97, 121105 (2018).

[58] In fact, naively taking $\tilde{\mathcal{C}}_{\mu}=-\tau_{R}^{-1} u_{\mu} \delta f_{q}$ in Eq. (6), one finds $\mathcal{A}_{\mu}=-\omega_{\mu}$. Nonetheless, the $\Sigma_{\mu}^{<(>)}$in $\mathcal{C}_{\mu}$ actually contains $\hbar$ corrections stemming from Wigner functions, which depend on the details of realistic collisions such as 2 to 2 scattering or the interactions with media. Such contributions hence make $\mathcal{A}_{\mu}$ undetermined without specifying a particular scattering process.
[59] Although CSE is known as a dual effect for CME in the vector/axial bases [3], it is automatically included in the CME currents in the right/left-handed bases.

[60] J.-H. Gao, Z.-T. Liang, Q. Wang, and X.-N. Wang, arXiv:1802.06216 [Phys. Rev. D (to be published)].

[61] K. Hattori and Y. Yin, Phys. Rev. Lett. 117, 152002 (2016).

[62] Y. Akamatsu and N. Yamamoto, Phys. Rev. Lett. 111, 052002 (2013). 